\section{Prostatakarzinom: Hormonentzug gefährdet auch periphere Gefäße}

Männer, bei denen aufgrund eines biochemischen Prostatakarzinomrezidivs die Sexualsteroidproduktion unterdrückt wird, sind besonders anfällig für koronare Herzerkrankung und Schlaganfall. Auch an den Arterien und Venen in der Peripherie scheint die Androgendeprivation nicht spurlos vorüberzugehen.

C teigen nach radikaler Prostatektomie oder Strahlentherapie eines nicht metastasierten Prostatakarzinoms die PSAWerte wieder an, ist die Androgendeprivation (ADT) der nächste therapeutische Schritt, meist medikamentös mit Agonisten oder Blockern des GonadotropinReleasing-Hormons (GnRH). Die Patienten müssen allerdings den günstigen Effekt des Hormonentzugs auf den Krebs mit einem höheren Risiko für eine koronare Herzkrankheit (KHK) oder einen Schlaganfall erkaufen. Ob auch das periphere Gefäßsystem unter dem Hormonmangel leidet, wollten nun US-amerikanische Wissenschaftler herausfinden.

Sie analysierten im Rahmen einer populationsbasierten Beobachtungsstudie die Krankenakten von über 182.757 Männern, die in der US National Institute Surveillance Epidemiology and End Re- sults (SEEER)-Medicare Datenbank gelistet waren. Die Teilnehmer waren 66 Jahre oder älter, wurden zwischen 1992 und 2007 mit ihrer Krebsdiagnose konfrontiert und litten an einem nicht metastasierten Prostatakarzinom. Sie hatten weder venöse Thromboembolien (VTE) noch Erkrankungen der peripheren Arterien in der Vorgeschichte. Während einer durchschnittlichen Nachbeobachtungszeit von 5,1 Jahren wurden 47,8\% der Teilnehmer mit GnRH-Analoga behandelt, 2,2 \% hatten sich einer Orchiektomie unterzogen.

Der Hormonentzug gefährdete tatsächlich auch die Gesundheit der peripheren Gefäße. So stieg unter ADT das Risiko einer peripheren arteriellen Verschlusskrankheit (PAVK) (adjustierte HR: 1,16; $95 \%$-CI: $1,12-1,21)$ sowie das einer venösen Thromboembolie (adjustierte HR:
1,10; 95\%-CI: 1,04-1,15) signifikant im Vergleich zu Männern, deren Sexualhormone nicht supprimiert worden waren. Andere Faktoren, die das PAVK- und VTE-Risiko ebenfalls negativ beeinflussen, hatten die Forscher in ihrer Auswertung bereits berücksichtigt.

Die beiden Gefäßsysteme scheinen allerdings unterschiedlich empfindlich auf den Hormonentzug zu reagieren: So stieg das PAVK-Risiko bereits nach ein bis vier Monaten ADT, das Risiko für VTE erst nach 13 Monaten.

Fazit: Die ADT bei Patienten mit nicht metastasiertem Prostatakarzinom erhöht das Risiko für eine PAVK oder VTE. Aufgrund der potenziellen Gefahren des Hormonentzugs fordern die Studienautoren, Nutzen und Risiken genauer zu erforschen, um diejenigen Patienten identifizieren zu können, die tatsächlich davon profitieren.

Dr.Dagmar Kraus

$\mathrm{Hu} \mathrm{JC}$ et al. Androgen-deprivation therapy for nonmetastatic prostate cancer is associated with an increased risk of peripheral arterial disease and venous thromboembolism. Eur Urol 2012; 61: 1119-28

\title{
Achtung:
}

\section{Hier muss der Dummy durch eine Anzeige ersetzt werden !!}

